\title{
SIMPLE PROOF FOR GOOFSPIEL: THE GAME OF PURE STRATEGY
}

\author{
MOSHE DROR, ${ }^{*}$ Ben Gurion University of the Negev
}

\begin{abstract}
A simple proof is given for the best strategy when the game of pure strategy is played.

STOCHASTIC DYNAMIC PROGRAMMING; BEST STRATEGY
\end{abstract}

Following Ross (1983), we examined the game of pure strategy referred to as Goofspiel and played by two players using a normal deck of cards. The description is taken almost literally from Ross (1983).

First, the 13 clubs are discarded. Of the remaining 39 cards, the 13 hearts are given to Player I, the 13 diamonds to Player II, and the 13 spades are placed face down in the center. The spades are shuffled and the top one is turned face up. At this point the two players choose one of their cards and then simultaneously discard them. The one who discards the higher card wins from the other an amount equal to the value of the upturned spade. In the case of a draw (both players discarded the same card) then neither wins, the three upturned cards are thrown away and a new spade is upturned. After 13 plays, there are no remaining cards and the game ends.

Consider the game under the assumption that Player II discards his cards in a random manner and that Player I knows that this random strategy was adopted by Player II. In this situation Ross proves that the best way for Player I to play is always to match the upturned spade. In a more general setting there are $N$ different cards $V_{1}, \cdots, V_{N}$ (instead of 13) having values $V_{1} \leqq V_{2} \leqq \cdots \leqq V_{N}$ (the hand of player I), simultaneously $Y_{1} \leqq Y_{2} \leqq \cdots \leqq Y_{N}$ (the hand of Player II), and $S_{1} \leqq S_{2} \leqq \cdots \leqq S_{N}$ ) the spades. The game is played as before.

Theorem. If Player II discards in completely random manner, then the strategy maximizing Player I's expected winning is the one that discards the card having value $V_{i}$ whenever the upturned spade has value $S_{i}, i=1, \cdots, N$.

Proof. Observe that given any two sets of numbers $A$ and $B$ of equal cardinality $A=\left\{a_{i}\right\}_{i=1}^{n}, B=\left\{b_{i}\right\}_{i=1}^{n}$ and let $\pi, \psi$ be any two permutations of $\{1,2, \cdots, n\}$. Denote $a_{\pi}=\left(a_{\pi(1)}, a_{\pi(2)}, \cdots, a_{\pi(n)}\right), b_{\psi}=\left(b_{\psi(1)}, b_{\psi(2)}, \cdots, b_{\psi(n)}\right)$ scalar product

$$
a_{\pi} \cdot b_{\psi}=\sum_{i=1}^{n} a_{\pi(i)} b_{\psi(i)}
$$

Then

$$
\max _{\pi, \psi} a_{\pi} b_{\psi}=\sum_{i=1}^{n} a_{i} b_{i}, \quad \text { where } \quad a_{1} \leqq a_{2} \leqq \cdots \leqq a_{n} ; b_{1} \leqq b_{2}<\cdots \leqq b_{n} .
$$

Given $N$ cards in the game, denote the probability that Player I wins given he discards the card $V_{i}$ by $P_{i}$. Clearly $P_{1} \leqq P_{2} \leqq \cdots \leqq P_{N}$. These probabilities are independent of the upturned value of the spade card $S_{i}$, and are non-increasing with $N$. Thus, the scalar product of the

Received 11 April 1989.

* Postal address: Industrial Engineering and Management, Ben Gurion University of the Negev, Beer Sheva, Israel.

Research partly carried out at the Centre de recherche sur les transports, Université de Montréal. 
ordered sequence of the spades vector (the $\left\{S_{i}\right\}_{j=1}^{N}$ ) with the win probabilities takes the maximal value when matched in order.

This optimal strategy proof does not use an induction argument or the interchange argument of the proof in Ross (1983).

Reference

Ross, S. M. (1983) Introduction to Stochastic Dynamic Programming. Academic Press, New York. 